\title{
LA COMPLEJIDAD DEL LENGUAJE EN LOS PROCESOS DE FACILITACIÓN DE GRUPOS
}

\section{THE COMPLEXITY OF LANGUAGE IN GROUP FACILITATION PROCESSES}

Fecha recepción: 15 de diciembre de 2019 / fecha aceptación: 20 de mayo de 2020

Rodrigo Severo Arce Rojas ${ }^{1}$

\section{Cómo citar este artículo:}

Arce, R. (2020). La complejidad del lenguaje en los procesos de facilitación de grupos. Revista Pensamiento y Acción Interdisciplinaria, 6(1), 90-107. DOI: http://doi.org/10.29035/pai.6.1.90

\section{Resumen}

El presente artículo de revisión es un producto derivado de la tesis doctoral del autor sobre facilitación y pensamiento complejo. Del análisis y síntesis se concluye que aunque ya existen enfoques y métodos de facilitación que abordan la complejidad éstos no han sido suficientemente difundidos y comprendidos por lo que aún predomina un enfoque de facilitación cartesiana que simplifica la realidad compleja. La realidad se presenta en sus dimensiones simples, complicadas y complejas y la facilitación cartesiana es más aplicable a situaciones simples pero tiene dificultades para abordar la complejidad que presenta fenómenos críticos, raros, inciertos, ambiguos, discontinuos y borrosos. Por más que facilitación tenga el propósito de hacer más fáciles las cosas se enfrenta con múltiples complejidades tales como la del cerebro, la mente, la inteligencia, la conciencia, el grupo, la comunicación, el lenguaje, entre otras complejidades. En este concierto de complejidades el presente artículo aborda la complejidad del lenguaje como parte de los procesos comunicacionales en la facilitación de grupos de diálogo. Se encuentra que la complejidad del lenguaje puede ser abordado desde los sistemas adaptativos complejos propios de las ciencias de la complejidad y el pensamiento complejo con sus principios de organización, dialogicidad, recursividad y hologramía. Como tal se entiende que el lenguaje es un sistema organizado que presenta un conjunto de elementos tangibles e intangibles que se encuentran interrelacionados e interdependientes que presentan la propiedad de autoorganización y emergencias, una dinámica no lineal alejada del equilibrio con capacidad de adaptación y evolución. Ello permita que se pueda generar la ampliación de la conciencia a partir de la resignificación de ideas iniciales producto del diálogo intersubjetivo. En tal sentido la facilitación de grupos puede ser enriquecido a partir del entendimiento de la complejidad del lenguaje.

Palabras clave: Complejidad, facilitación, grupos, interculturalidad, lenguaje.

1 Peruano, Ingeniero Forestal, Doctor en Pensamiento Complejo, Universidad Ricardo Palma, Lima, Perú. Correo electrónico: rarcerojas@yahoo.es 


\begin{abstract}
This review article is a derivative of the author's doctoral thesis on facilitation and complex thinking. From the analysis and synthesis, it is concluded that although there are already approaches and methods of facilitation that address complexity, these have not been sufficiently disseminated and understood, so a Cartesian facilitation approach that simplifies complex reality still predominates. Reality is presented in its simple, complicated and complex dimensions and Cartesian facilitation is more applicable to simple situations but has difficulties in addressing the complexity that presents critical, rare, uncertain, ambiguous, discontinuous and blurred phenomena. As much as facilitation has the purpose of making things easier, it faces multiple complexities such as that of the brain, mind, intelligence, consciousness, group, communication, language, among other complexities. In this concert of complexities the present article addresses the complexity of language as part of the communicational processes in dialogue group facilitation. It is found that language complexity can be approached from the complex adaptive systems characteristic of the complexity sciences and complex thought with its principles of organization, dialogicity, recursion and hologram. As such, it is understood that language is an organized system that presents a set of tangible and intangible elements that are interrelated and interdependent and that present the property of self-organization and emergence, a non-linear dynamic far from equilibrium with the capacity for adaptation and evolution. This allows the expansion of consciousness to be generated from the resignification of initial ideas resulting from intersubjective dialogue. In this sense, group facilitation can be enriched by understanding the complexity of language.
\end{abstract}

Key words: Complexity, facilitation, groups, interculturality, language.

\title{
Introducción
}

Aunque cada vez la facilitación de grupos adopta enfoques y métodos de complejidad (como por ejemplo los diálogos apreciativos, la búsqueda de futuro, espacios abiertos y café global, entre otros) todavía la comunidad de practicantes mantiene enfoques de lo que se podría llamar facilitación cartesiana dominada por el paradigma del pensamiento simplificante de la ciencia positivista. Esta actitud es reforzada por el hecho que una de las formas de definir la facilitación es hacer fáciles las cosas y reducir la complejidad de la realidad.

Pero el tema es que los grandes problemas que afronta la humanidad son de la naturaleza compleja tales como el cambio climático, la deforestación, la pérdida de diversidad biológica, las pandemias, la pobreza, la corrupción, entre otras, en los que los enfoques disciplinares o multidisciplinares no son suficientes. La facilitación en un mundo cada vez más complejo requiere por tanto desarrollar nuevos enfoques, métodos y técnicas que sean capaces de afrontar la complejidad de la realidad.

Los grupos humanos en diálogo están conformados por personas con capacidad de autoorganización y el rol de la facilitación es permitir fluir la emergencia 
de la resignificación de sus posiciones iniciales y encontrar nuevos sentidos a partir de la ampliación de la conciencia (De la Mata, 2013). La facilitación enfrenta múltiples complejidades tales como la mente, la inteligencia, la conciencia, los grupos humanos, la sociedad, la comunicación y el lenguaje, entre otros. En este contexto es importante ponderar la complejidad del lenguaje en la dinámica no lineal de los encuentros grupales orientados a aclarar un tema, ponerse de acuerdo, explorar posibilidades, elaborar un plan o estrategia, entre los diferentes propósitos de la facilitación. El estudio de la complejidad del lenguaje resulta fundamental en todo proceso comunicacional más allá del intercambio de significados inmediatos o perceptibles.

La realidad finalmente está conformada por materia/masa, energía, información y sentido. La información que adopta múltiples formas es central en la naturaleza. Es en este marco que se entiende que la comunicación y lenguaje están estrechamente interrelacionados aunque la comunicación no se reduce al lenguaje oral o escrito. Otras formas de comunicación tienen que ver con expresiones físicas o químicas. El fenómeno de la comunicación humana es abordado desde diferentes disciplinas y su comprensión requiere un enfoque de investigación interdisciplinaria y transdisciplinaria.

El entendimiento del lenguaje puede ser abordado por el enfoque de los sistemas adaptativos complejos propios de las ciencias de la complejidad como desde el pensamiento complejo en su arista más filosófica (Arce, 2017). Los sistemas adaptativos complejos están conformados por múltiples y heterogéneos elementos que están interrelacionados, son interdependientes e interdefinibles. Estos sistemas presentan las propiedades de autoorganización y de emergencias que son manifestaciones distintas a los comportamientos individuales de sus constituyentes. Estos sistemas presentan una dinámica no lineal que quiere decir que no necesariamente existe proporcionalidad entre las causas y los efectos y existe una alta sensibilidad a las condiciones iniciales. Asimismo estos sistemas tienen la capacidad de comunicarse con el entorno con el que establecen relaciones de mutua influencia. Estos sistemas tienen la capacidad de aprendizaje y evolución. Se caracterizan además porque son irreductibles. Adicionalmente estos sistemas son de control difuso (acéntricos o policéntricos) (Maldonado 2001, 2003, 2005, 2009, 2011, 2001a, 2013, 2014, 2014a, 2016; García, 2006).

Por su parte, el pensamiento complejo busca entender y comprender la realidad de todo aquello que escapa a la ciencia normal para poder contribuir a su transformación, a través de la confluencia de una diversidad formas de pensar (pensamiento crítico, pensamiento sistémico/relacional, pensamiento divergente/ convergente, pensamiento lateral, pensamiento arborescente, pensamiento filosófico, entre otros). El pensamiento complejo, ampliamente desarrollado por Morin (1998, 1999, 2001, 2002, 2004, 2008) y ampliado por otros, como por ejemplo Rodríguez \& Aguirre (2011), Luengo (2016), ofrece el marco epistemológico para abordar la realidad desde el paradigma de la complejidad o la realidad 
imbricada y no descomponible y se reconocen los principios de organización, dialogicidad, recursividad y hologramía para la comprensión de la realidad. No es una negación o subestimación del paradigma cartesiano que todo lo fragmenta sino una perspectiva integradora en la que hay que saber moverse entre el caos y el orden pues la realidad muchas veces se aleja del equilibrio y otras veces busca orden y dirección.

El presente artículo de revisión está orientado a contribuir con el entendimiento de la complejidad del lenguaje para que inspire su adopción en los procesos de facilitación de diálogo en los grupos y así ampliar su radio de acción centrado fundamentalmente en una lógica de simplificación. Facilitar no es negar la sencillez pero tampoco es evadir la complejidad de la realidad con todas sus interrelaciones, incertidumbres, ambigüedades y borrosidades.

Aunque ya ha sido abordado el tema de la complejidad del lenguaje desde la perspectiva de la lingüística, la comunicación, la psicología, entre otras disciplinas el tema de tratarlo como tema específico de la facilitación de grupos es algo más novedoso. Se espera que inspire investigaciones científicas más sistemáticas para que este apasionante campo del lenguaje pueda ser útil para la facilitación efectiva de procesos de diálogo grupal.

\section{Marco referencial}

\section{Complejidad}

La complejidad trata fundamentalmente de los fenómenos impredecibles, inestables, raros, irregulares con el fin de indeterminar, desequilibrar, incrementar grados de libertad y abrir posibilidades. La complejidad trata de las crisis y convive con las incertidumbres, azares, aleatoriedades, brumas, borrosidades, cambios súbitos, catástrofes, y bifurcaciones, entre otras propiedades. A diferencia del pensamiento que divide la complejidad es un pensamiento de síntesis. (Maldonado 2014, 2016)

La realidad, o mejor dicho las realidades, tienen su propia complejidad, lo que no quiere decir que todas las cosas sean complejas pues también hay expresiones simples y complicadas. Las realidades simples poseen pocas variables y este caso funcionan mejor las relaciones lineales de causa y efecto, el determinismo y la predictibilidad. Las realidades complicadas pueden estar conformadas por varios elementos pero la característica fundamental es que son descomponibles y su abordaje requiere un enfoque técnico (Alaimo \& Salías, 2015). La figura 1 muestra las diferentes formas como se presenta la realidad o los fenómenos y describe sus características centrales y las herramientas que se usan para su estudio. La 
realidad compleja puede a su vez ser desorganizada y organizada y esta última puede a su vez ser de complejidad creciente (Rodríguez \& Aguirre, 2011).

Figura 1: Diferentes formas como se presenta la realidad o los fenómenos

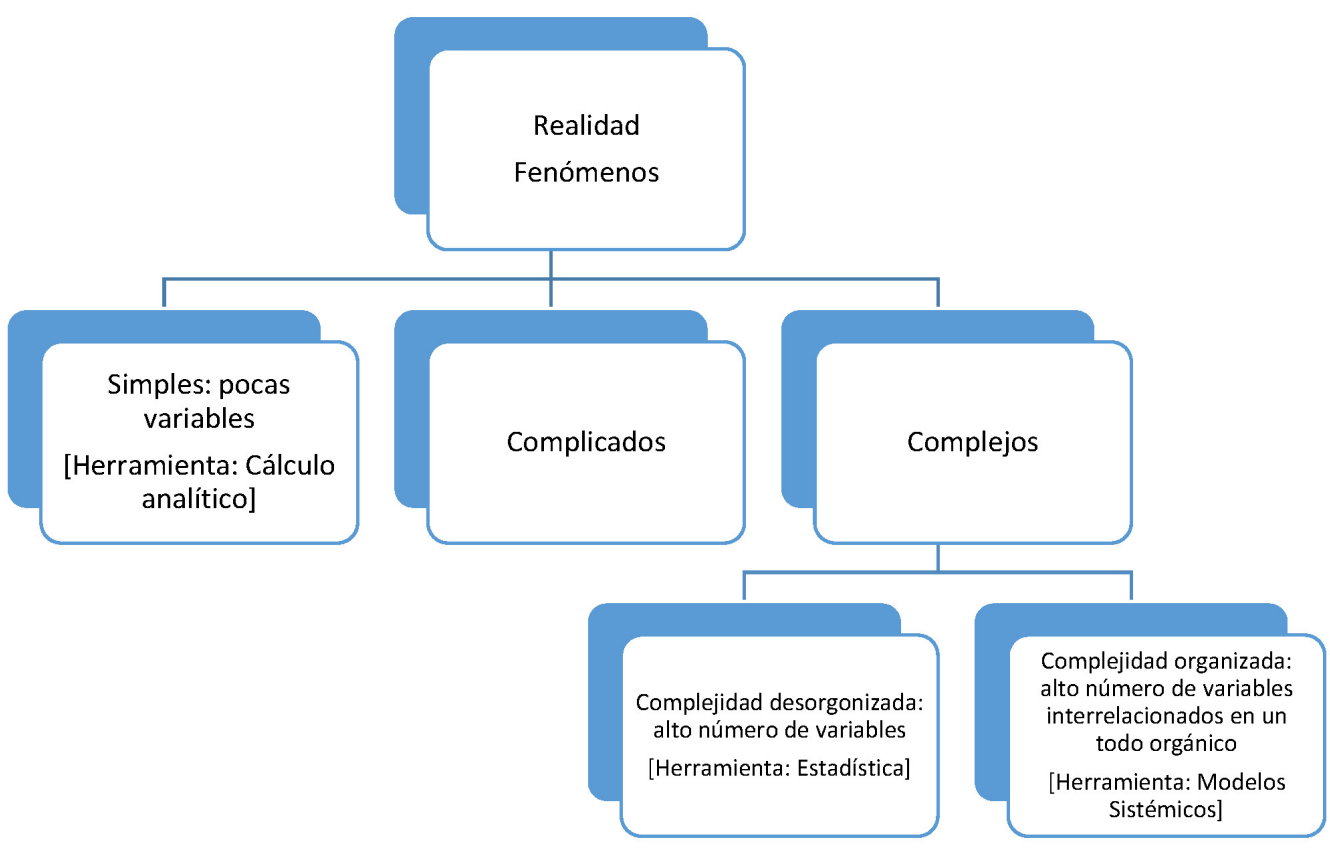

Fuente: Rodríguez \& Aguirre, 2011.

\section{Lenguaje}

El lenguaje puede ser descrito desde la perspectiva de la complejidad (Mora, 2018). Zamorano (2012) reconoce a la comunicación verbal humana como un sistema complejo porque es un proceso, no un acto, presenta comportamiento difícilmente predecible, presenta emergencia sistémica, es un sistema fuera del equilibrio y presenta autoorganización, entre otras propiedades. Castro \& Flórez (2007) añaden los atributos de fases transicionales, sensibilidad, estabilidad, variabilidad y no linealidad, entre otros. Las lenguas, como el resto de los sistemas complejos, se caracterizan por ser dinámicas, escalables y sin un "control central" (Johnson 2007, p.15).

El lenguaje no es solo una herramienta que permite comunicarse sino que también permite categorizar (Ellis, 1992), representar e intercambiar conceptos a nivel social (Arce, 2005; Piedra, 2010) y construir significados. Meyin (1981) indica que el lenguaje desempeña un papel activo en la estructuración de la conciencia humana. Según Mora (2018, p. 3) "el lenguaje está cargado de sentidos y signos, pero a la vez tiene una función afectiva e imaginativa, con la cual podemos 
construir modelos de la realidad". Como apunta Piedra (2010, p. 17) "el lenguaje está íntimamente ligado al pensamiento, a la expresión de ideas, a la creatividad y a la cognición en general mediante una relación co-articulada y co-evolutivamente desarrollada". El lenguaje puede presentarse como sistema, como facilitador de formas específicas de relación y acción sobre el medio y como fenómeno concreto (Colombo, 2008).

El lenguaje no sólo está constituido por la comunicación verbal pues también cuenta la comunicación no verbal y el espacio y distancia que emplean los interlocutores en el proceso de interacción comunicacional. Así se dice que la comunicación es un proceso de interacción entre los signos, la semiótica y la proxémica (Arango, 2009).

\section{Facilitación}

Tradicionalmente la facilitación es concebida como los procesos y funciones que permiten que un grupo humano pueda alcanzar sus objetivos en un ambiente agradable y haciendo buen uso de los recursos y el tiempo (Instituto de Estudios Ambientales, 1993). Arce (2018, p. 35) define la facilitación:

...como procesos y funciones que permiten interactuar con los paradigmas, pensamientos, sentimientos, emociones y manifestaciones (discursos, actitudes y prácticas) de personas y colectivos de modo tal que se favorezca el despliegue del conjunto de sus capacidades, facultades y potencialidades orientado a que alcancen un equilibrio interno o un equilibrio social, siempre dinámicos, para establecer relaciones, vínculos, entendimientos o acuerdos que eventualmente se pueden convertir en objetivos que deriven a acciones transformadoras.

El cuadro 1 muestra las diferencias entre una facilitación cartesiana y una facilitación con capacidad de abordaje de la complejidad. 
Cuadro 1: Diferencias entre una facilitación cartesiana y una facilitación con capacidad de abordaje de la complejidad.

\begin{tabular}{|l|l|}
\hline \multicolumn{1}{|c|}{ Facilitación cartesiana } & $\begin{array}{l}\text { Facilitación con capacidad de abordaje } \\
\text { de la complejidad }\end{array}$ \\
\hline Sistema cerrado & Sistema abierto \\
\hline Lineal & No lineal \\
\hline Determinista & Indeterminista \\
\hline Directivo & Autoorganizado \\
\hline Simplifica & Afronta la complejidad \\
\hline Busca certezas & Afronta las incertidumbres \\
\hline $\begin{array}{l}\text { Se fundamenta en los significados inmediatos } \\
\text { de las palabras }\end{array}$ & Reconoce la complejidad del lenguaje \\
\hline
\end{tabular}

Sin embargo, hay que reconocer, que estas dos formas de facilitación no son absolutamente polarizadas sino que se producen entrecruzamientos mutuos de tal manera que se ajusta más a los sistemas caórdicos (Figueroa, 2016; Abad \& Sánchez, 2009). Ya existen métodos que abordan la complejidad que requieren ser mejor comprendidos, practicados y desarrollados para que incrementen su capacidad de afrontar la complejidad de la realidad. Una de estas perspectivas es la que aborda la facilitación sistémica (Fuks, 2009).

El cuadro 2 muestra las diversas metodologías de facilitación y su efectividad con relación a la complejidad reducida y elevada complejidad. 
Cuadro 2: Diversas metodologías de facilitación y su efectividad con relación a la complejidad reducida y elevada complejidad

\begin{tabular}{|l|l|l|}
\hline \multicolumn{1}{|c|}{$\begin{array}{c}\text { Metodología de } \\
\text { facilitación }\end{array}$} & \multicolumn{1}{c|}{$\begin{array}{c}\text { Aplicación en } \\
\text { complejidad reducida }\end{array}$} & $\begin{array}{c}\text { Aplicación en elevada } \\
\text { complejidad }\end{array}$ \\
\hline Diálogos apreciativos & $* * *$ & $* *$ \\
\hline Laboratorio de cambio & $* *$ & $* *$ \\
\hline Círculo de prosperidad & $* *$ & $* *$ \\
\hline Democracia profunda & $* *$ & $* * *$ \\
\hline Búsqueda de futuro & $*$ & $* *$ \\
\hline Escuela de Paz \\
Palestino-Israelí & $* * *$ & $* * *$ \\
\hline Espacios abiertos & & $* * *$ \\
\hline Planificación por escenarios & $*$ & $* *$ \\
\hline Diálogo sostenido & $*$ & \\
\hline Café global & $* *$ & \\
\hline
\end{tabular}

Fuente: Mille et al. 2008

\section{Grupo}

Un grupo puede ser concebido como un sistema en el que al menos dos personas establecen interacciones de influencia (Shaw, 1983; Peñuela \& Álvarez, 2005; Blanco et al., 2005;) En esta perspectiva es importante el concepto de plus grupal (el todo es más que la suma de las partes) (Soto, 2003; Valeria, 2015;). Cada persona en el grupo llega con sus paradigmas, creencias, estereotipos, representaciones sociales, imaginarios; convicciones, certezas; preguntas, inquietudes, búsquedas; sueños, proyecciones; posiciones, intereses, necesidades; referencias, antecedentes, historia, entre otros elementos que son exteriorizados en discursos, narrativas y textos y dan riqueza al diálogo. Pero no solo son objetividades las que están presentes sino, sobre todo, el proceso de diálogo da cuenta de las interrelaciones entre subjetividades que se ponen de manifiesto a través del lenguaje verbal y no verbal. 


\section{Aspectos metodológicos}

El presente artículo de revisión derivado de la tesis doctoral del autor sobre facilitación y pensamiento complejo para la Multiversidad Mundo Real Edgar Morin. En el desarrollo de la tesis se realizó una investigación bibliográfica sobre la facilitación, se analizaron las relaciones entre la facilitación y la complejidad a partir del análisis y síntesis de la Teoría de grupos de la psicología social, la Teoría de los sistemas sociales de la sociología y la Teoría de la comunicación de las ciencias de la comunicación. Se realizó entrevistas a profundidad a expertos facilitadores iberoamericanos y se presentaron los resultados a un grupo focal de expertos para poner en consistencia los resultados hallados (Arce, 2018). En dicha investigación se acotó el estudio de la complejidad a la dinámica de los grupos mientras que este artículo se da énfasis a la complejidad del lenguaje. Para los propósitos de este artículo se ha complementado con revisión bibliográfica especializada sobre complejidad, lenguaje, facilitación y grupo, se realiza una discusión integrada y se extraen concusiones.

\section{Resultados y Discusiones}

Aunque la facilitación lleva consigo la idea de hacer más fáciles las cosas para que un grupo humano pueda establecer acuerdos o explorar opciones se enfrenta a la complejidad del mundo. En tanto la facilitación involucra personas entonces confluye la complejidad de la vida, la complejidad del grupo, del cerebro, de la conciencia, de la comunicación, del lenguaje, entre otras complejidades (Arce, 2018). Por tanto la pretensión de hacer más fáciles las cosas no puede descuidar la complejidad de la realidad o incluso de las múltiples realidades. Descuidar estas complejidades Ileva a una facilitación reduccionista que puede tener la apariencia de ser efectiva aun cuando sea ampliamente celebrada.

Las interacciones de las múltiples complejidades aludidas tienen una manifestación tangible en el lenguaje pero no como algo acabado sino como una expresión temporal de tendencia en tanto cada palabra o frase emitida puede ser parte de una cadena de significados jerarquizados y anidados en sistemas que interactúan de manera implícita. Así, una cosa es lo que se dice y otra cosa es lo que se pretende decir o lo que se dice entre líneas. Asimismo una cosa es lo que se dice y otra cosa es la que se entiende porque el proceso de decodificación comunicacional no siempre es lineal y está sujeta siempre a los intereses y perspectivas del interlocutor. Ello es posible porque el lenguaje muchas veces es polisémico y ambiguo.

En los procesos de diálogo se requiere más capacidad de apertura, capacidad para explorar otras posibilidades y no quedarse únicamente con el patrón de referencia con el que se cuenta. El reto de la facilitación es que el propio proceso 
dialógico vaya generando significados, sensibilidades y sentidos compartidos. Aunque se parta de significados diferentes es la interacción dinámica la que va encauzando una corriente de significación común que es lo que permite ir convergiendo en acuerdos o en todo caso dejando claras las diferencias. Tan importante como alcanzar los consensos es reconocer los disensos porque permite definir los campos de actuación conjunta o simplemente identificar puntos en los que no es posible construir agendas compartidas sin que ello signifique fractura de la relación, empatía o los afectos.

Es importante reconocer que el complejo proceso de construcción de sentido en la experiencia humana está basado en los mecanismos de internalización y externalización de significados que se presentan como procesos recursivos. Todos estos procesos y transiciones ocurren durante la experiencia en el contacto con el mundo al mismo tiempo que se siente, se piensa y se actúa (Fossa, 2017). De ahí la importancia de no quedarse en las externalización de significados sino también reconocer el diálogo interno, pues como afirma Shanon (2008) el punto de partida de la comunicación humana es la subjetividad de la consciencia.

Aunque se puede mencionar que en el grupo todos son iguales y que todos tienen la oportunidad de expresarse esto puede ser una sobre simplificación de una realidad en la que efectivamente existen diferencias de recursos y oportunidades. Si no se toma en cuenta la diversidad, inocentemente, los facilitadores pueden legitimar una serie de injusticias como las epistemológicas y de lenguaje y terminan siendo funcionales a los grupos de poder.

Para favorece las expresiones de diversidad de los grupos es necesario considerar el tema de la representatividad social, cultural, de género y edad. Por cuestiones prácticas muchas veces se prefiere invitar al proceso de diálogo solo a los actores formales o visibles. Es importante considerar que mucho de los ausentes tienen mucho que decir y al invisibilizarlos se pierden voces que podrían tener elementos cruciales para el proceso de diálogo formal (Santos, 2002).

Además, es de especial importancia reconocer los ámbitos en los que se desenvuelve la facilitación. En el caso de procesos grupales que dirige el Estado se encuentra que el lenguaje está fuertemente inscrito en marco estratégico (políticas públicas, normas) y sus funciones y competencias. En nombre del Estado de Derecho no es posible salirse de un campo predeterminado de significados. Lo mismo sucede en el campo organizacional privado que está fuertemente influenciado por una visión globalizante, mercadocéntrica, de crecimiento económico, eficiencia, productividad y competitividad. En ambos espacios se ve que hay una fuerte adscripción a un modelo único y acabado de desarrollo y todo proceso de diálogo no puede salirse de ese sistema cerrado. Por ello es que cuando se entra en procesos de diálogo con otros actores hay dificultades para el entendimiento y establecimiento de acuerdos. Se verifican procesos de encuentro en que ceden más los actores con cosmovisiones distintas que el Estado o las empresas porque 
se asume que sus argumentos son más fuertes, contundes y objetivos en tanto se basan en evidencias científicas, aunque no siempre esto es cierto. En un mundo de verdades únicas y una realidad dominante entonces no hay mucho pie al reconocimiento de las múltiples verdades y los pluriversos (Arce, 2019).

En casos donde existan marcos culturales distintos se requiere una facilitación intercultural respetuosa de las diversas cosmovisiones y ontologías puesto que existen diversas formas de relacionarse con la naturaleza. La ontología relacional implica el reconocimiento de esas diversas formas de concebir la relación entre los seres humanos y la naturaleza en el que hay cabida a la concepción de la vida que involucra humanos, no humanos e incluso lo que convencionalmente se considera como elementos inertes pero que para muchas culturas también tienen vida propia como los ríos o las montañas (Escobar, 2005, 2014). Frente a una facilitación cartesiana donde se concibe el lenguaje como algo plano y uniforme es necesario asumir los diversos marcos ontológicos y epistémicos que tienen influencia en los diversos significados con los que actúan los interlocutores. Al respecto, Noam Chomsky (citado por Littlejhon, 1987) define el lenguaje como una estructura que cambia de acuerdo al contexto cultural. Por tanto, hay palabras y conceptos claves que requieren ser explicitados para favorecer un proceso de diálogo mucho más respetuoso y horizontal. En estos casos, puede ser que antes que homogeneizar conceptos lo más conveniente sería que se visibilicen las diferencias y desarrollar el diálogo sobre ese marco.

En un proceso comunicacional existe al menos dos dimensiones, una dimensión que alude a lo perceptible y está más ligado a los significados literales de las palabras, y una dimensión subyacente que puede ser soterrada como la comunicación no verbal, los elementos de poder, rango, los énfasis y tonos del lenguaje, entre otros. Pero tampoco estas dimensiones van absolutamente paralelas y separadas porque en ocasiones una dimensión aflora sobre la otra como cuando se dice una palabra que luego provoca arrepentimiento o simplemente aparece como discordante. En psicología se habla de actos fallidos.

De ahí la necesidad de incorporar a la facilitación la identificación de los paradigmas que son las ideas centrales que gobiernan los pensamientos, sentimientos, emociones y las diversas manifestaciones humanas tales como los discursos y las narrativas (Ventrella, 2014). Los paradigmas también pueden ser reconocidos como las creencias, lentes o maneras de ver las cosas que influyen sobre el lenguaje y comportamientos de las personas. Los paradigmas no son uniformes pues dependen de la medida en que cada actor lo ubica en una jerarquía de modos de ver el mundo o las propias prioridades que tiene el actor. Hasta ahora se ha privilegiado en la facilitación de grupos la administración de los discursos inmediatos pero no se ha explorado a profundidad los paradigmas. En tal sentido, la identificación de paradigmas es una competencia clave de los facilitadores. 
Aunque los facilitadores teóricamente deben permanecer imparciales, reconocido como el principio de la neutralidad, esto es difícil lograrlo porque la sola presencia del facilitador influye en la dinámica de los grupos. Desde la teoría de la comunicación todo comunica, los discursos, los gestos, los énfasis, los silencios e incluso las ausencias comunican e influyen en la objetividad del grupo (Watzlawichk, Beavin \& Jackson, 1971). Ello implica que el facilitador debe honestamente reconocer su posición explícita o implícitamente según sea el caso para que la emergencia de significados y sentidos del grupo sea hasta donde sea posible producto de la interacción e intersubjetividad del grupo.

Actualmente, en una era dominada por las tecnologías de la información se encuentran los siguientes hechos:

- Hay mayor cantidad de información y cobertura a la diversidad de información que profundización de los significados y sentidos de la información.

- La ingente cantidad de información no siempre se traduce en mayor dedicación a la lectura y comprensión.

- Existe una tendencia a la simplificación del lenguaje y a la sobrevaloración de las imágenes, los íconos y los emoticones.

- Existe libre circulación de falsedades que por acumulación se convierten en verdades (posverdades).

Todos estos fenómenos son importantes tomarlos en cuenta en la facilitación de grupos porque el fenómeno de la comunicación no se reduce a la dinámica dialógica del ambiente en el que se encuentran y existe permanente interacción con la información que se trae desde la historia personal y colectiva así como con la abundante información del entorno. El lenguaje es producto de un sistema abierto cuyas manifestaciones forman parte una interrelación sistémica que moviliza lo interno como lo externo y forma parte de los encadenamientos entre individuo-sociedad-especie y cuerpo-mente-fisiología-palabra-acción-medio. El lenguaje pone de manifiesto la cultura pero a su vez la cultura influye en los significados y sentidos del lenguaje. El lenguaje que se usa en los grupos da cuenta a la vez de los procesos de comunicación que se generan desde los medios, desde la educación y todo espacio de socialización real o virtual. Es así que es posible reconocer cómo los principios del pensamiento complejo están presentes en el lenguaje de los grupos: la propia organización del lenguaje, la dialogicidad, la recursividad y la retroacción entre significados y la hologramía. Esto puede ser descrito graficando la complementación o diferencias de significados, la capacidad de que los significados se retroalimenten o se influyan mutuamente, el hecho que significado esté en el individuo como miembro del grupo y el significado que emerge del grupo se manifieste en el individuo. Así es posible apreciar cómo el lenguaje es un fenómeno complejo. 
Existen múltiples formas cómo es posible que el diálogo sea más enriquecedor para lograr que el proceso de facilitación del grupo no se quede con la información inicial con el que se empieza el diálogo sino que se brinden las oportunidades para tener un proceso profundamente reflexivo para así poder avanzar a un estadio más propositivo. Por ejemplo se podría convenir los significados clave que están en la discusión sin que sea un proceso de transmisión de la información sino de formulación conjunta cuando eso es posible. En otros casos se puede hacer visible las diferencias de concepción y hacerlos más explícitos para que sean tomados en cuenta y se entienda desde qué marcos de referencia se está expresando. Es necesario cuidar que en el proceso de diálogo no se produzcan injusticas epistemológicas o lingüísticas.

Una de las herramientas clave del facilitador es la pregunta y se requiere apelar permanentemente a ella para invitar al grupo revisar y profundizar sobre sus ideas, ponerlas en cuestión, analizar implicancias posibles de una decisión, analizar impactos de no tomar una decisión sin que ello signifique apurar una decisión que terminen imponiendo sutil o abiertamente posiciones hegemónicas desde posiciones de poder.

Es importante identificar los paradigmas que gobiernan las ideas, sentimientos y discursos de los interlocutores, en contextos de interculturalidad es necesario explicitar los marcos epistémicos y ontológicos y evitar aplastar sentidos y sentires en nombre del acuerdo acelerado. Por cuestiones de tiempo y pragmatismo en ocasiones los facilitadores permiten una decisión rápida para avanzar en el proceso pero no se miden las consecuencias. En este marco la calidad de la facilitación no está en qué medida se logran acuerdos rápidos sino la medida en que estos acuerdos respetan las ontologías, son justas, equitativas y contribuyen genuinamente con la sustentabilidad.

Aunque tanto la facilitación cartesiana como la facilitación con capacidad de afrontar la complejidad generan emergencias que se traducen en significados compartidos, se estima que la diferencia estaría en la calidad de la ampliación de la expansión de la conciencia y la intensidad del compromiso con el significado mutuamente reconocido. En la medida que una facilitación sistémica ha logrado movilizar todo el ser, con toda su historia, su contexto y sus sensibilidades profundas habría un mayor compromiso con la sustentabilidad de los acuerdos. Esta premisa se sustenta en el hecho que la conciencia y la intencionalidad son hechos biológicos que permiten los actos de habla y que existe una relación entre el lenguaje y la intencionalidad colectiva como hecho necesario para la constitución de hechos institucionales (Sánchez, 2017). 


\section{Conclusiones}

Aunque ya existen enfoques y métodos de facilitación que abordan la complejidad éstos no han sido suficientemente difundidos y comprendidos por lo que aún predomina un enfoque de facilitación cartesiana que simplifica la realidad compleja. La realidad se presenta en sus dimensiones simples, complicadas y complejas y la facilitación cartesiana es más aplicable a situaciones simples pero tiene dificultades para abordar la complejidad que presenta fenómenos críticos, raros, inciertos, ambiguos, discontinuos y borrosos. Por más que facilitación tenga el propósito de hacer más fáciles las cosas se enfrenta con múltiples complejidades tales como la del cerebro, la mente, la inteligencia, la conciencia, el grupo, la comunicación, el lenguaje, entre otras complejidades. En este concierto de complejidades el presente artículo aborda la complejidad del lenguaje como parte de los procesos comunicacionales en la facilitación de grupos de diálogo. Se encuentra que la complejidad del lenguaje puede ser abordado desde los sistemas adaptativos complejos propios de las ciencias de la complejidad y el pensamiento complejo con sus principios de organización, dialogicidad, recursividad y hologramía. Como tal se entiende que el lenguaje es un sistema organizado que presenta un conjunto de elementos tangibles e intangibles que se encuentran interrelacionados e interdependientes que presentan la propiedad de autoorganización y emergencias, una dinámica no lineal alejada del equilibrio con capacidad de adaptación y evolución. Ello permita que se pueda generar la ampliación de la conciencia a partir de la resignificación de ideas iniciales producto del diálogo intersubjetivo. 


\section{Referencias bibliográficas}

Abad, Y., \& Sánchez, L. (2009). Un estudio con análisis factorial de componentes principales en la apreciación de los vecinos de los asuntos ciudadanos del Municipio Bolívar (Tesis licenciatura). Universidad de Oriente, Barcelona España.

Alaimo, M., \& Salías, M. (2015). Proyectos ágiles con Scrum. Buenos Aires: Kleer.

Arango, C. (2009). Ideas para ir de la semiótica del signo a la semiótica del discurso en el texto audiovisual: Un mínimo itinerario nocional. Revista Luciérnaga Audiovisual, 2(2), 51-62.

Arce, M. (2005). Las huellas del zapatero. Costa Rica: Alambique.

Arce, R. (2017). La Facilitación del Diálogo desde una Perspectiva de Complejidad. Pensamiento del Sur, 1, 4-12.

Arce, R. (2018). Aportes del pensamiento complejo a los enfoques, metodologías y la práctica de facilitación de procesos sociales (Tesis Doctoral). Multiversidad Mundo Real Edgar Morin, México. México.

Arce, R. (2019). Pensamientos emergentes orientados al reconocimiento del pluriverso. Recuperado de https://www.servindi.org/actualidadopinion/30/04/2019/pensamientos-emergentes-orientados-alreconocimiento-del-pluriverso.

Blanco, A., Caballero, A., \& de la Corte, L. (2005). Psicología de los grupos. Madrid: Pearson.

Castro, J., \& Flórez, R. (2007). La emergencia del lenguaje y los sistemas dinámicos. Revista Colombiana de Psicología, 16, 185- 202.

Colombo, M. (2008). Lenguaje. Una introducción al estudio psicológico de las habilidades humanas para significar. Buenos Aires, Eudeba.

De la Mata, G. (2013). Metodologías de Inteligencia Colectiva: La técnica del espacio abierto. Recuperado de http://innovationforsocialchange.org/metodologiasde-inteligencia-colectiva-la-tecnica-del-espacio-abierto-open-space/absolut.

Ellis, J. (1992). Language, thought and logic [Lenguaje, pensamiento y lógica]. Evanston, III: Northwestern University Press.

Escobar, A. (2005). El "postdesarrollo" como concepto y práctica social. En D. Mato (Coord.), Políticas de economía, ambiente y sociedad en tiempos de globalización. (pp.17-31). Caracas: Universidad Central de Venezuela.

Escobar, A. (2014). Sentipensar con la tierra. Nuevas lecturas sobre desarrollo, territorio y diferencia. Medellín: UNAULA. 
Figueroa, C. (2016). Trabajo en red y sistemas de articulación colaborativos. Madrid: Tejeredes.

Fossa, P. (2017). Pleromatización, fisionomización y metaforicidad: una articulación teórica de los procesos de construcción de sentido de Valsiner, Werner y McNeill. Psicología USP, 28(1), 93-101.

Fuks, S. (2009). La Facilitación Sistémica de Procesos Colectivos: una artesanía de contextos orientada hacia la creatividad y la participación colaborativa. Buenos Aires: Universidad Nacional de Rosario- Fundación Moirü.

García, R. (2006). Sistemas complejos. Conceptos, método y fundamentación epistemológica de la investigación interdisciplinaria. Barcelona: Gedisa.

Instituto de Recursos Mundiales y Grupo de Estudios Ambientales, A. C. (1993). El proceso de evaluación rural participativa. Una propuesta metodológica. Cuaderno N¹. México: Programa de Manejo Participativo de los Recursos Naturales.

Johnson, N. (2007): Simply Complexity, a clear guide to complexity theory. Londres: Oneworld.

Littlejohn, S.W. (1987) Theories of human communication. California: Wadsworth Publishing Company.

Luengo, E. (2016). Las vertientes de la complejidad. Diferencias y convergencias. Congreso Mundial por el Pensamiento Complejo. Los desafíos en un mundo globalizado. Congreso llevado a cabo en París, Francia.

Maldonado, C. (Ed.). (2001). Visiones sobre la complejidad. 2ạ ed. Bogotá: Universidad El Bosque.

Maldonado, C. (2003). El problema de la filosofía del conocimiento y el estudio de los sistemas complejos. Praxis Filosófica, 17, 103-120.

Maldonado, C. (2005). Ciencias de la complejidad: Ciencias de los cambios súbitos. En Universidad Externado de Colombia (Ed), Odeón: Observatorio de Economía y Operaciones Numéricas (pp. 85-125). Bogotá: Universidad Externado de Colombia.

Maldonado, C. (2009). Complejidad de los Sistemas Sociales: Un reto para las ciencias sociales. Cinta Moebio, 36, 146-157.

Maldonado, C., \& Gómez, N. (2011). El Mundo de las Ciencias de la Complejidad. Una investigación sobre qué son, su desarrollo y sus posibilidades. Bogotá: Universidad del Rosario.

Maldonado, C. (2011a). Termodinámica y complejidad. Una introducción para las ciencias sociales y humanas. Bogotá: Desde abajo. 
Maldonado, C. (2013). Significado e impacto social de las ciencias de la complejidad. Bogotá: Desde Abajo.

Maldonado, C. (2014). ¿Qué es un sistema complejo? Revista. Colombiana de Filosofía y Ciencia, 14(29), 71-93.

Maldonado, C. (2014a). Reflexión sobre las implicaciones políticas de la complejidad. ALPHA, 38, 197-214.

Maldonado, C. (2016). El evento raro. Epistemología y complejidad. Cinta moebio, 56, 187-196.

Meyin, M. (1981). Lenguaje e identidad cultural en sociedades coloniales. (Tesis de maestría). Universidad de Puerto Rico, San Juan, Puerto Rico.

Mille, M., Roehl, H., Knuth, M., \& Magner, C. (2008). Trazando el diálogo. Herramientas esenciales para el cambio social. Bilbao: Taos Institute Publications, Fundación Gizagune.

Mora, A. (2018). Las concepciones sobre el lenguaje y su relación con los procesos cognitivos superiores, en docentes de I Ciclo y II Ciclo de Educación General Básica de escuelas públicas urbanas de tres cantones de la provincia de San José, Costa Rica. Revista Educación, 42(1), 20.

Morin, E. (1998). Introducción al pensamiento complejo. Barcelona: Gedisa.

Morin, E. (1999). Los 7 saberes necesarios para la educación del futuro. Paris: Organización de las Naciones Unidas para la Educación, la Ciencia y la Cultura.

Morin, E. (2001). El método 1: La naturaleza de la naturaleza. Madrid: Cátedra.

Morin, E. (2002). Los principios generativos y estratégicos del método. En E, Morin, E, Ciurana \& R. Motta. Educar en la era planetaria. El pensamiento complejo como método de aprendizaje en el error y en la incertidumbre humana. México: Universidad de Valladolid.

Morin, E. (2004). El Método, Tomo 6. La Ética. Paris: Seuil.

Morin, E. (2008). El año I de la era ecológica. Barcelona: Paidos.

Peñuela A., \& Álvarez, L. (2005). Complejidad: Grupos y Procesos de Comunicación. Razón y Palabra, 46(10), Recuperado de http://www.razonypalabra.org.mx/ anteriores/n46/penuelalvarez.html.

Piedra, L. (2010). Deixis personal y representaciones mentales: Propuesta de la existencia de los marcadores deícticos cognitivos y su relación con la deixis personal. (Tesis de maestría). Universidad de Costa Rica, San Juan, Costa Rica. 
Rodríguez, L., \& Aguirre, L. (2011). Teorías de la complejidad y ciencias sociales. Nuevas Estrategias Epistemológicas y Metodológicas. Nómadas. Critical Journal of Social and Juridical Sciences, 30(2), 147-166.

Sánchez, J.S. (2017). Conciencia, intencionalidad y lenguaje: el reconocimiento agencial como base de la ontología social. Cuadernos de Filosofía Latinoamericana, 38(117), 175-210.

Santos, B. (2002). Para uma sociología das ausencias e uma sociología das emergencias. Revista Crítica de Ciencias Sociais, 63, 237-260.

Shanon, B. (2008). The representational and presentational: an essay on cognition and the study of mind. Charlottesville: Imprint Academic.

Shaw, M. (1983). Dinámica de grupo. Psicología de la conducta de los pequeños grupos. Barcelona: Herder.

Soto, M. (2003). Apuntes sobre psicología social y grupos. Anuario 2002. México: UAM-X.

Valeria, F. (2015). Los dispositivos grupales y su uso pedagógico. Una reflexión sobre el grupo operativo y la multiplicación dramática Enseñanza e Investigación en Psicología, 2(20), 196-205. Recuperado de http://www.redalyc.org/articulo. oa?id=29242799010.

Ventrella. S. (2004). El poder del pensamiento positivo en las empresas. Bogotá: Norma.

Watzlawick, P. Beavin, J., \& Jackson, D. (1971). Teoría de la Comunicación Humana. Interacciones, Patologías y Paradojas. Buenos Aires: Tiempo Contemporáneo.

Zamorano, A. (2012). Teorías del caos y lingüística: aproximación caológica a la Comunicación Verbal Humana. Revista Signa 21, 679-705.

Dirección de correspondencia:

Rodrigo Severo Arce Rojas

Ingeniero Forestal, Doctor en Pensamiento Complejo, Universidad Ricardo Palma, Lima, Perú.

Contacto: rarcerojas@yahoo.es

Esta obra se encuentra bajo una Licencia de Creative Commons

Reconocimiento-NoComercial-Compartirlgual 4.0 Internacional 